\title{
MIRANDO MÁS ALLÁ DE LAS FRONTERAS. LA EXPERIENCIA DE LAS PIONERAS AFROAMERICANAS
}

\author{
LOOKING BEYOND THE FORNTIERS. THE EXPERIENCE OF THE PIONEER \\ AFRO-AMERICAN WOMEN
}

María Luz Arroyo Vázquez

UNED - Madrid

\section{RESUMEN:}

Este artículo pretende profundizar en las vidas de dos mujeres que fueron de granimportancia en la lucha contra la discriminación racial en Estados Unidos: Rosa Paks y Coretta Scott King. Sus hechos e iniciativas marcaron un antes y un después en la historia de este país.

\section{Palabras claves:}

Derechos civiles, Rosa Parks, Coretta Scott King.

\section{Abstract:}

This article aims to go deeper into the lives of two women who were extremely important in the fight against racial discrimination in the United States: Rosa Parks and Coretta Scott King. Their actions and initiatives marked a before and an after in the History of their country.

\section{KeY WORD:}

Civil laws, Rosa Parks, Coretta Scott King. 
“The only tired I was, was tired of giving in" Rosa Parks

"How many men must die before we can really have a free and true and peaceful society?" Coretta Scott King

\section{INTRODUCCIÓN}

Esta comunicación aborda la experiencia de las pioneras afroamericanas, centrándose en el ejemplo de Rosa Parks y Coretta Scott King como mujeres representativas de todas aquéllas que abrieron nuevos espacios en la lucha por los Derechos Civiles en los Estados Unidos. Asimismo, debemos destacar que la labor relevante de estas dos activistas contra la segregación racial tuvo eco dentro y fuera de las fronteras estadounidenses.

Tanto Rosa Parks como Coretta Scott King figuran entre las mujeres más influyentes de los Estados Unidos en el siglo XX, pues se considera que su lucha fue esencial para conseguir la abolición de un marco legal que permitía la segregación racial. El acto de valentía de Rosa Parks, que se negó a obedecer la orden de un conductor de autobús de ceder su asiento a un pasajero blanco en la ciudad de Montogomery (Alabama) en 1955, fue la chispa que llevó a impulsar el Movimiento por los Derechos Civiles en los Estados Unidos, con la convocatoria de un boicot contra el servicio de autobuses que, después de resistir 381 días, terminó con la segregación racial en el transporte público y desarticuló la segregación institucionalizada en el Sur de los Estados Unidos.

Por su parte, Coretta Scott King ya se había interesado por la defensa de los derechos civiles antes de conocer a su marido, Martin Luther King Jr. Sin embargo, esta relación marcó toda su vida, pues a partir de entonces caminó junto a él y manifestó su oposición a la segregación racial. Continuó en esa misma línea tras el asesinato de su esposo, en 1968. Queremos destacar que estas pioneras compartieron un sueño, la necesidad de mejorar las relaciones interraciales y las condiciones sociales de todos los seres humanos.

\section{ABRIENDO NUEVOS ESPACIOS EN LA LUCHA POR LOS DERECHOS CIVILES} 2.1. Rosa Parks (1913-2006)

Rosa Louise McCauley, conocida como Rosa Parks, nació en Tuskegee (Alabama) en 1913. Era hija de un carpintero y una maestra. Estudió en la Montgomery Industrial School for Girls y en el Alabama State Teachers College. Se graduó en el instituto, cuando sólo el 7\% de las personas de color lo lograban. Después de terminar sus estudios se casó con Raymond Parks, con quien trabajó para abolir la opresión racial. Ambos se unieron a la National Association for the Advancement of Colored People
(NAACP) (Asociación Nacional para el Progreso de la Gente de Color), para tratar de conseguir que los derechos civiles de los negros fuesen respetados.

Antes del incidente del autobús, Rosa Parks había mostrado su firme determinación de querer cambiar las cosas que consideraba injustas. En 1943, empezó a trabajar como secretaria local en la NAACP. Seis años más tarde se convirtió en asesora de la asociación, donde trabajó de forma activa en favor de los derechos civiles. También había mostrado su activismo con su asistencia al Highlander Folk School, un centro educativo, para defender los derechos de los obreros y la igualdad racial. En Alabama, uno de los estados con mayor discriminación de los Estados Unidos, el autobús era un escenario más de la incesante y degradante segregación existente en barrios, restaurantes y lugares públicos, igual que ocurría en el resto de los estados sureños de los Estados Unidos desde el final de la guerra civil. El 1 de diciembre de 1955, concretamente en Montgomery (Alabama), Rosa Parks volvía a casa desde su trabajo en la sección de corte y confección de unos grandes almacenes. Ya había sido expulsada del autobús en más de una ocasión, pero esta vez todo se desarrolló de forma distinta. Al subir, ella notó que el conductor, James Blake, era el mismo hombre que la había forzado a bajar del autobús unos doce años antes. Aquella vez el fondo del vehículo estaba lleno de gente, de modo que ella se había subido por la parte delantera y, por esa razón, el conductor la había echado. La costumbre era que los blancos se situasen delante y los negros, detrás. Si no había asientos suficientes para los blancos, los afroamericanos tenían que levantarse y cederles los suyos. El conductor de autobús no había cambiado en doce años, pues pidió a los pasajeros negros que se levantaran y dejaran libres los asientos que habían ocupado para que se sentaran los blancos. Las otras tres personas negras se levantaron, pero Rosa Parks permaneció inmóvil, pues, según declara en su autobiografía, cuanto más cedían peor se les trataba (Parks, 1992: 115). Rosa Parks ya había padecido suficiente intimidación, estaba cansada del maltrato que los afroamericanos recibían, harta del racismo y de los estatutos sureños, denominados Jim Crow Laws, que eran leyes que imponían la segregación. En varias ocasiones hizo hincapié en que no era verdad que estuviese físicamente cansada, sino harta de ceder (Parks, 1992: 116). Pensaba que ese trato no era justo, idea que le habían transmitido personas a las que ella admirada, como su madre y sus abuelos. Rosa Parks relata que fue su madre la persona que la educó para que fuera fuerte y quien le enseñó la importancia del respeto, la libertad y la igualdad: "My mother was a teacher in a little school, and she believed in freedom and equality for people, and did not have the notion that we were supposed to live as we did, under legally enforced racial segregation. She didn't believe in it" (Interview, June 2, 1995: 1).

En esa misma línea, Rosa Parks describe perfectamente la situación discriminatoria que vivió en Montgomery durante su infancia, adolescencia y 
juventud, explicando que existía una completa segregación racial permitida y reforzada por las leyes, y que luchó contra ello, pues sentía que no era justo verse privada del derecho a la libertad (Interview "Standing Up for Freedom", 1995). En una entrevista concedida a la BBC a la edad de 90 años, Rosa Parks afirmaba:

No importaba cuál fuera tu ingreso, tu educación o tu profesión. Si eras negro, debías vivir en un barrio para negros. 40 años después esto ya no es así. Era fácil identificar el racismo entonces. Se veía cada día. Se hablaba de eso en las casas, en las comunidades, en las iglesias, en las escuelas. Siempre estaba con uno. Todovía hay racismo en Estados Unidos, pero las formas de racismo no son tan obvias como hace 40 años (BBC Mundo, 28 de agosto de 2003: 3).

Rosa Parks fue arrestada, encarcelada y condenada a pagar 10 dólares de multa más 4 dólares de gastos por el juicio, pues según las leyes de segregación racial su conducta era ilegal. La legislación en vigor en 1955 señalaba que las personas de piel negra debían ceder el asiento a la población blanca. Además, no podían ocupar las primeras filas en los transportes públicos, pese a que el importe del billete era el mismo para cualquier viajero. La National Association for the Advacement of Colored People se ocupó del caso y pagó una fianza para que Rosa Parks saliera de la cárcel, a pesar de no haber considerado otros casos semejantes sucedidos poco antes, pues supuestamente no eran idóneos para ejercer una presión legal. Parks fue la tercera mujer detenida en pocos meses, tras Mary Louis Smith y Claudette Colvin. Seguramente, en su caso debió influir el hecho de que fuera una empleada de la NAACP. Diez años antes, la asociación también había ofrecido ayuda legal a Irene Morgan y había obtenido una victoria en el Tribunal Supremo, terminando así con la segregación racial en el comercio interestatal.

La detención de Rosa Parks despertó la indignación en la población afroamericana de Montgomery y tuvo como efecto la formación de la Montgomery Improvement Association (Asociación por el Desarrollo de Montgomery) para defender los derechos civiles de los negros. El grupo eligió como uno de sus portavoces a un joven pastor de la Iglesia Bautista desconocido hasta entonces, Martin Luther King Jr. Se organizó el boicot contra el servicio de autobuses, que comenzó el lunes 5 de diciembre, el día del juicio de Rosa. Jo Ann Robinson y otras mujeres de la Women's Political Council habían escrito unas octavillas cuyas ideas fueron utilizadas para propagar el modo de protesta. Treinta mil afroamericanos que previamente habían sido clientes de los autobuses actuaron de manera solidaria. En lugar de viajar en autobús, caminaban o se juntaban para viajar en coche. Un afroamericano propietario de una empresa de taxis también ayudó a los viajeros bajando sus tarifas hasta igualarlas a las de los autobuses. Este movimiento no violento fue fuerte y no sucumbió, a pesar de las vicisitudes y las amenazas. La solidaridad de la gente siguió inamovible y sacudió la conciencia de la sociedad estadounidense, cuya actividad llegó a ser conocida en el resto del mundo.

Un año más tarde, tras 381 días de boicot a los autobuses, hubo una respuesta. El Tribunal Supremo declaró que la segregación en el transporte público iba en contra de la Constitución estadounidense. Dos años antes, el 17 de mayo de 1954, el Tribuna Supremo había dictado una declaración de inconstitucionalidad contra la segregación racial en la escuela pública, al considerar que permitía que existiese la desigualdad. Rosa Parks afirma que muchos pensaron entonces que esa misma idea podía ser aplicada a otras cuestiones como el transporte (Parks, 1992: 100). El Movimiento por los Derechos Civiles tuvo un efecto en los políticos, a pesar de no cambiar la mentalidad de muchos blancos sureños (Parks, 1992: 167). El Presidente demócrata Lyndon Baines Johnson, nacido y criado en Texas, firmó unas leyes claves: el 2 de julio de 1964, una ley prohibió la discriminación racial en los lugares públicos y más tarde, el 6 de agosto de 1965, la Voting Rights Act prohibía las prácticas discriminatorias adoptadas en muchos estados sureños, tales como los literacy tests, que trataron de impedir el derecho de los negros a votar. A partir de ese momento, el movimiento por los derechos civiles cobró un tremendo ímpetu en el largo camino hacia la igualdad de derechos para los afroamericanos. En la entrevista concedida a BBC Mundo en 2003, cuando se le preguntó si ella habría imaginado que su acto de coraje tendría tal impacto, Rosa Parks contestó:

No tenía idea de que alguien se enteraría de lo que me había sucedido aque día Ni siquiera tenía la certeza de que sobreviviría a aquel día. Simplemente estaba cansa del maltrato. El joven blanco que estaba de pie no hab́́a pedido estabia pedido estaba sentada donde se suponia que debí hacelo. Elconductor exigió a cuatro personas negras que se pusieran de pie por una persona blanca que no habia pedido un asiento. Simplemente sentí que no podía permitirme seguir siendo maltratada de esa manera (BBC, agosto de 2003)

Poco después de la decisión del Tribunal Supremo de abolir la discriminación en el transporte público, en 1957 Rosa Parks se trasladó a Hamptom (Virginia) y posteriormente a Detroit (Michigan), donde prosiguió su actividad en defensa de los derechos civiles. Participó en varias manifestaciones fundamentales, como la que tuvo lugar en 1963 en Washington para impulsar las leyes federales sobre derechos civiles. Entre 1965 y 1988 trabajó como recepcionista y auxiliar de oficina para el representante demócrata del Congreso, John Conyers. En 1987 Rosa Parks creó el Rosa and Raymond Parks Institute for Self Development junto a su amiga Elaine Eason Steele. El instituto fue fundado en honor de su marido Raymond, que había muerto de cáncer en 1977, para ayudar a los jóvenes a alcanzar su máximo potencial con programas que les educasen sobre su pasado, su presente y su futuro. El centro patrocinó el programa anual Pathways 
to Freedom (Caminos hacia la Libertad) y organizó recorridos en autobús para dar a conocer a los jóvenes la historia de la lucha por los derechos civiles en los Estados Unidos. Rosa Parks tuvo trato con muchos líderes políticos, viajó y recibió muchos premios y honores en reconocimiento a su esfuerzo por conseguir la armonía racial. Entre los premios que obtuvo destacan la Medalla Presidencial de la Libertad, que recibió en 1996, y la Medalla de Oro del Congreso, que le entregó el Presidente Bill Clinton en 1999. El Museo y la Biblioteca de Rosa Parks le fueron dedicados en noviembre de 2001 y más de cincuenta universidades le concedieron el doctorado Honoris Causa. En el mes de agosto de 1994 Parks fue atacada en su domicilio de Detroit por un joven afroamericano, Joseph Skipper, que quería dinero. El incidente causó sorpresa, cuando Parks confesó haber preguntado al joven antes de que le atacase si sabía quién era ella ante lo cual él le respondió que no sabía quién era, pero que tampoco le importaba.

Rosa Parks se mantuvo activa hasta el final de sus días como una incansable defensora de los derechos civiles y se ha convertido en un icono de la defensa de la libertad y la igualdad racial de todos los seres humanos. No obstante, pocos años antes de morir, afirmó que, a pesar de los logros, en los Estados Unidos todavía quedaba un largo camino por recorrer para mejorar las relaciones raciales. Rosa Parks murió el 24 de octubre de 2005, a los 92 años de edad. Se celebró una ceremonia especial en su honor y fue la segunda persona en ser homenajeada en la Rotonda del Congreso. Cabe destacar su sencillez, al considerar que no había cambiado las cosas sola, sino que hubo más gente como ella y que ella fue sólo una más entre las muchas personas que lucharon por la libertad.

\subsection{Coretta Scott King (1927-2006)}

Coretta Scott King fue, sin duda alguna, una de las mujeres afroamericanas más influyentes en la sociedad estadounidense del siglo XX y gozó de una gran proyección internacional. Su capacidad de luchar de manera incansable por los derechos civiles al tiempo que transmitía un mensaje de no violencia y de respeto por los Derechos Humanos tuvo eco en todos los lugares del mundo. Nació y se crió en una zona rural del sur en la granja de su abuelo, situada a doce millas de Marion (Alabama). Tuvo una infancia sacrificada, pues ayudaba a su familia en las tareas del campo, y participó en la recolección de algodón desde muy temprana edad. Desde pequeña fue consciente de la injusticia social que suponía la segregación racial, al verse privada de los derechos que le correspondían: "When I was very young and growing up, I was protected from the extreme hardships of segregation though I was always aware of being deprived of the rights to which I was entitled" (King, 1969: 28). Así, por ejemplo, tenía que caminar varios kilómetros para poder asistir a la escuela, mientras que los niños blancos iban en autobús a otros colegios más cercanos. Vivía en una comunidad en la que todos eran negros. No obstante, se sentía afortunada por vivir en una granja grande de la que su familia había sido propietaria durante más de tres generaciones, y afirmaba que ese factor le hizo sentir orgullo, autoestima y dignidad (King, 1969: 29). Cuando terminó sus estudios en el Lincoln High School en 1945, su fue a estudiar con una beca al Antioch College, en Yellow Springs, Ohio. Allí comenzó su interés por el movimiento a favor de los derechos civiles y se unió a la NAACP y a los comités universitarios Race Relations y Civil Liberties. Al finalizar sus estudios de música y educación, consiguió una beca para estudiar canto en el New England Conservatory of Music de Boston, donde se graduó en canto y en violín. Allí conoció a Martin Luther King Jr., con quien se casó en junio de 1953 y tuvo cuatro hijos.

En 1954, el matrimonio King se trasladó a Montgomery (Alabama), donde Martin Luther King había aceptado el puesto de pastor en la Dexter Baptist Church. Allí, en 1955, comenzaría el boicot a los autobuses al que nos hemos referido anteriormente. Dr. Martin Luther King se convirtió en el gran protagonista y adquirió un papel de liderazgo en la organización de las distintas formas de protesta. Coretta afirma que, afortunadamente, aconsejado por ella y por su madre, Martin no aceptó el puesto de presidente de la NAACP cuando se le ofreció en noviembre de 1955, ya que entonces no podría haber aceptado el liderazgo en el boicot de los autobuses (King, 1969: 111). Según Coretta, las peores facetas de la segregación en Montgomery eran las reglas de las líneas de autobuses. Ella nos describe con rigurosa exactitud cómo, a pesar de que los negros constituían el $70 \%$ de los viajeros, eran tratados peor que el ganado: Of all facets of segregation in Montgomery, the most degrading were the rules of the Montgomery City Bus Lines. This northern-owned corporation outdid the South itself. Although seventy percent of its passengers were black, it treated them like cattle-worse than that, for nobody insults a cow" (King, 1969: 111).

Una de las iniciativas de Coretta para apoyar firmemente a su marido consistió en actuar en los Conciertos por la Libertad queella describe comola historia dela lucha desde 1955 a 1965 contada en prosa y canción (King, 1969: 248). Con ellos trataba de conseguir fondos para la Southern Christian Leadership Conference (SCLC), la organización que había fundado y presidía Martin Luther King Jr. Dio su primer concierto por la libertad el 14 de noviembre de 1964 en el New York City's Town, con el que consiguió llenar el auditorio y una recaudación de 6.000 dólares. Con los conciertos se reunieron grandes fondos para el SCLC y sus afiliados, lo cual fue un motivo de satisfacción para Coretta. Asimismo, solía acompañar a su marido en las manifestaciones que tenían lugar en distintas ciudades. En algunas ocasiones, como en el caso de la marcha de Washington de 1963, en la que Martin dio el famoso discurso "I Have a Dream", no pudo estar en la primera fila junto a él. Curiosamente, Rosa Parks menciona que no se permitía que las mujeres tuviesen un papel relevante y que el Comité organizador de la Marcha no 
quería que Coretta o las esposas de los otros líderes marcharan junto a sus maridos, por lo que quedaron relegadas a permanecer en un segundo plano. Según el programa, las mujeres tampoco hablarían, pero sí se concedió un pequeño tributo a aquéllas que habían participado en la lucha por los derechos civiles, como Josephine Baker, Marian Anderson y Lena Horne, que fue la única que pronunció la palabra "libertad". De hecho, las mujeres negras sufrían una doble discriminación, racial y de género. Rosa declara en su autobiografía que las mujeres quedaban en un segundo término y que por aquel entonces la defensa de sus derechos aún no se había convertido en una causa generalizada (Parks, 1992: 166). En otras ocasiones, Coretta sí que pudo estar en primera fila, como en la marcha desde Selma hasta Montgomery (Alabama) el 7 de marzo de 1965

Dado que Martín Luther King Jr. participaba en continuas manifestaciones y sentadas de protesta, se vio detenido y encarcelado en varias ocasiones, lo cual afectaba profundamente a su familia. Así, en 1960 estuvo en la cárcel de Atlanta y en diciembre de 1961, en Albany. Durante su estancia en la prisión de Reidsville, el senador John F. Kennedy, que se encontraba en los últimos días de su campaña para la presidencia de los Estados Unidos, llamó a Coretta y, tras manifestarle su preocupación por su marido, le brindó su ayuda, que ella aceptó de buen grado. Martin salió de la prisión de Reidsville en libertad bajo fianza. Coretta afirmó que la intervención de Kennedy en el caso de su marido resultó decisiva para ganar la presidencia:

\begin{abstract}
John Kennedy was elected President of the United States by only about a hundred thousand votes. It is my belief that historians are right when they say that his
intervention in Martin's case won the presidency for him. That seemed significant to intervecause of what happ of the Kennedys to what Martin was trying to accomplish (King, 1969: 197).
\end{abstract}

Visitó diversos países con su marido: Ghana, con motivo de la independencia del país en 1957, México en 1958, y la India en 1959, en un peregrinaje para conocer los lugares asociados con Mahatma Gandhi, que tanto les había influido con su filosofía de la no violencia. Sin duda, uno de los viajes más significativos fue el que realizaron a Oslo en 1964 para que Martín Luther King Jr. recibiese el premio Nobel de la Paz. Ella explica en su autobiografía lo orgullosa que se sintió al escuchar el discurso de Martin Luther King Jr., que, al aceptar el premio, manifestó con humildad que le pertenecía a toda la gente que formaba parte del Movimiento por los Derechos civiles: "I was proud of Martin and what he stood for. I was proud that his work, and that of his associates, had made better the lives of our countrymen. I was proud that black people all over the world had felt renewed courage and hope because of this man, my husband" (King, 1969: 13). El compromiso para mantener la paz fue una de las causas más importantes para Coretta. De este modo, en 1962 asistió como delegada de la Women's Strike for Peace a un congreso sobre el desarme en Ginebra (Suiza). En 1967 se manifestó contra la guerra de Vietnam e hizo de vínculo entre organizaciones que estaban a favor de la paz.

Después del asesinato de Martin Luther King Jr. en 1968, en Memphis (Tennessee), Coretta aumentó su actividad por los derechos civiles y su lucha en favor de las minorías, y alcanzó un mayor protagonismo en el plano político y social. Alzó su voz en nombre de los derechos de los niños y de las mujeres, de la dignidad de los homosexuales, del derecho al empleo, a la sanidad y a la educación, del desarme nuclear y del respeto del medio ambiente.

En el discurso que dio en el Memphis City Hall el 8 de abril de 1968, manifestó su deseo de continuar con la tarea que había iniciado junto a su marido, haciendo hincapié en los logros que se habían alcanzado desde aquel boicot que tuvo lugar doce años atrás en Alabama. Al mismo tiempo, expresó el camino que faltaba por recorrer, ya que, además de obtener demandas de tipo político, los negros aspiraban a alcanzar poder económico:

We must carry on because this is the way he would have wanted to have been. We are not going to get bogged down. I hope in this moment we are going to go forward; we are going to continue his work to make all people truly free and to make every person feel that he is a human being. His campaign for the poor must go on. Twelve years ago in Montgomery, Alabama, we started out with the bus protest, trying to get a seat, the right to sit down on the bus in any seat that was available. We moved through that period on to the period of desegregating public accommodations and on through voting rights, so that we could have political power. And now we are at the point where we must have economic power (King, 1969: 346).

En 1969 Coretta empezó a buscar apoyo para la construcción del Martin Luther King Jr. Center for Nonviolent Social Change en Atlanta (Georgia), un monumento a la memoria de su marido. El Centro King funciona como museo, institución educativa y alberga la mayor colección de documentos sobre el Movimiento a los Derechos Civiles. Otra de las metas que Coretta persiguió, para lo que realizó una gran campaña, fue la de conseguir que la fecha del nacimiento de Dr. King se convirtiese en festividad nacional, lo cual se legisló en 1983 y se celebró por primera vez en 1986.

Coretta tuvo la oportunidad de conocer a importantes personalidades espirituales tales como el Papa Juan Pablo II y el Dalai Lama y dialogó con figuras clave del mundo de la política, con diferentes jefes de estado, primeros ministros y presidentes norteamericanos, como Lyndon B. Johnson o Bill Clinton. Durante los años ochenta Coretta protestó de forma enérgica contra las políticas segregacionistas de Sudáfrica y llegó incluso a ser arrestada junto a sus tres hijos en la embajada de Sudáfrica en Washington D.C. en 1985. Viajó hasta Sudáfrica para apoyar 
a Winnie y Nelson Mandela, que entonces era un preso político. También presionó para que se aplicasen sanciones contra Sudáfrica. En 1994 Coretta estuvo presente cuando Nelson Mandela fue elegido democráticamente primer Presidente de Sudáfrica. Otro de los aspectos destacados de la labor de Coretta Scott King fue su defensa de las coaliciones interraciales, como las que creó en 1974 y en 1983. En 1974 formó una amplia coalición que comprendía más de 100 organizaciones religiosas, laborales, de negocios y de defensa de los derechos de la mujer. En 1983 consiguió que se unieran más de 800 organizaciones en defensa de los derechos humanos para formar la Coalition of Conscience en el vigésimo aniversario de la Marcha de Washington, hasta aquel entonces la mayor manifestación que había tenido lugar en la capital. En 1988 volvió a reunir la Coalition of Conscience, en el vigésimo quinto aniversario de la Marcha de Washington. Consciente de que, aunque la situación en la que se hallaba la población negra había mejorado, aún quedaban miedos e intimidaciones a las que se debía hacer frente, en 1987 la primera dama de los Derechos Civiles ayudó a dirigir la Mobilization Against Fear and Intimidation en Forsyth County (Georgia). En los años noventa participó en numerosos acontecimientos políticos, entre los que destaca su asistencia, invitada por el Presidente Bill Clinton, a los Acuerdos de Paz de Oriente Próximo, donde pudo presenciar el histórico apretón de manos entre Isaac Rabin y Yasir Arafat.

En 2003 se pronunció en contra de la invasión de Irak y permaneció activa en la defensa de la igualdad racial y social, y de la paz, hasta su muerte en enero de 2006. Dedicó especial atención a la lucha contra el sida y la educación para la no violencia ante el alarmante ascenso del uso de las armas en los Estados Unidos de América.

\section{CONCLUSIÓN}

Rosa Parks y Coretta Scott King desempeñaron un papel clave en la lucha contra la discriminación racial en la historia de los Estados Unidos. El gesto de valor de Rosa Parks fue decisivo para el Movimiento por los Derechos Civiles, pues indujo a cambiar los hilos de la historia estadounidense al desencadenar un boicot contra el servicio de autobuses, que culminó con la decisión del Tribunal Supremo de acabar con la segregación institucionalizada. A partir de ahí, se allanó el camino en la adopción de una legislación fundamental para acabar con las políticas y las prácticas discriminatorias. Por su parte, Coretta Scott King, que llegó a ser conocida como "la primera dama del movimiento por los derechos civiles", no sólo compartió el sueño de su marido de terminar con la segregación sino que, tras el asesinato del Dr. King, hizo que el sueño perviviera y, con su protesta, contribuyó a hacerlo realidad más allá de la frontera estadounidense, como ocurrió en el caso de Sudáfrica.

En definitiva, Rosa Parks y Coretta Scott King fueron mujeres firmemente comprometidas con la defensa de la libertad y la igualdad, y plantearon a la sociedad preguntas claves como la que formuló Coretta en su discurso en Memphis tras el asesinato de su marido: ¿Cuántos hombres deben morir antes de que realmente podamos tener una sociedad pacífica, auténtica y libre? ¿Cuánto tiempo se tardará? ("How many men must die before we can really have a free and true and peaceful society? How long will it take?") (King, 1969:347).

\section{REFERENCIAS BIBLIOGRÁFICAS}

Hare, K., "The Story of the Montgomery Bus Boycott", Montgomery Advertiser, November 5, 2005

Humphrey, J. L. y Nasca, F. L. N., “The Difference Made by One Person: Celebrating Rosa Parks", Curriculum Review, vol. 43, 4 diciembre 2003.

Parks, R. y Haskins, J., Rosa Parks: My Story, New York, Dial Books, 1992.

Scott King, C., My Life with Martin Luther King Jr., New York, Holt Rinehart and Winston, 1969.

“Washington honra a Rosa Parks", El País, 30 de octubre de 2005, p. 45.

"Rosa Parks", The Washington Times, 30 de octubre de 2005, B02.

"Rosa Parks Spurns Image Awards", The Washington Times, 16 de marzo de 2003, B05.

“Washington honra a Rosa Parks”, El País, 30 de octubre de 2005, p. 45. 